\title{
High-Temperature Thermodynamic Properties of Forsterite
}

\author{
PHIIIPPE GILLET
}

Laboratoire de Minéralogie Physique, Université de Rennes I

PASCAL RICHET AND FRANÇOIS GUYOT

Institut de Physique du Globe, Paris

GUILLAUME FIQUET

Laboratoire de Minéralogie Physique, Université de Rennes I

\begin{abstract}
The high-temperature thermodynamic properties of forsterite were reviewed in the light of a new determination of the isobaric heat capacity $\left(C_{p}\right)$, up to $1850 \mathrm{~K}$, and Raman spectroscopic measurements, up to $1150 \mathrm{~K}$ and $10 \mathrm{GPa}$. The $C_{p}$ measurements and available data on thermal expansion $(\alpha)$ and bulk modulus $(K)$ show that the isochoric specific heat $\left(C_{v}\right)$ exceeds the harmonic limit of Dulong and Petit above $1300 \mathrm{~K}$. This intrinsic anharmonic behavior of $C_{v}$ can be modeled by introducing anharmonic parameters $a_{i}=\left(\partial \ln v_{i} / \partial T\right)_{V}$ which are calculated from the measured pressure and temperature shifts of the vibrational frequencies. These parameters are all negative, with absolute values lower for the stretching modes of the $\mathrm{SiO}_{4}$ tetrahedra $\left(a_{i}=-1 \times 10^{-5} \mathrm{~K}^{-1}\right)$ than for the lattice modes $\left(a_{i} \approx-2 \times 10^{-5} \mathrm{~K}^{-1}\right)$. Through the relation $C_{p}=C_{v}+\alpha^{2} K_{T} V T$, the calculated anharmonic $C_{v}$ and the measured $C_{p}$ are then used to determine the temperature dependences of the thermal expansion and bulk modulus of forsterite, up to $2000 \mathrm{~K}$, in agreement with recent experimental results. Finally, all these data point to an inconsistency for the Grüneisen parameter of forsterite, whereby the macroscopic parameter $\gamma=\alpha V K_{T} / C_{v}$ cannot be evaluated simply at high temperature by summation of the individual isothermal mode Grüneisen parameters $\gamma_{i T}=K_{T}\left(\partial \ln v_{i} / \partial P\right)$.
\end{abstract}

\section{INTRODUCTION}

A great many mantle minerals are not amenable to thermodynamic measurements because of their instability at moderate temperatures or of the minute amounts of material that can be synthesized. To setup thermodynamic models of mantle phase equilibria, use is thus made of spectroscopically determined heat capacities and entropies [e.g., Kieffer, 1979]. The difficulty with this approach, however, is that there are virtually no measurements for testing the validity of the method under the high-temperature conditions of the mantle.

Forsterite is probably the mineral that has been studied the most extensively. The purpose of this contribution is thus to consider forsterite as a model mineral to investigate the consistency of the thermodynamic and spectroscopic data from ambient up to high pressures and temperatures. Our purpose is not to model mineral properties in the most comprehensive way, as done for example in the lattice dynamics study of forsterite by Rao et al. [1988], and the reasons for our simplified approach are twofold. First, experiments like inelastic neutron scattering are not feasible on minerals for which thermodynamic measurements are lacking. Thus we will consider only spectroscopic data that can be gathered on highpressure phases. Second, lattice-dynamics calculations are still

Copyright 1991 by the American Geophysical Union.

Paper number $91 \mathrm{JB} 00680$

0148-0227/91/91JB-00680\$05.00 done within the framework of the quasi-harmonic approximation whose limitations at high temperatures must be assessed. As a matter of fact, the role of anharmonicity can be investigated rather simply from the limited spectroscopic information that can be obtained for mantle minerals [Gillet $e$ t al., 1989].

In this paper we are mainly interested in the isobaric $\left(C_{p}\right)$ and isochoric $\left(C_{v}\right)$ heat capacities, thermal expansion coefficient $(\alpha)$ and isothermal bulk modulus $\left(K_{T}\right)$ of forsterite. Through calculations of the heat capacity from spectroscopic data, we first show that anharmonicity contributes significantly to $C_{v}$ and the other thermodynamic properties of forsterite above $1300 \mathrm{~K}$. Then we discuss the consistency of the available $C_{p}, \alpha$ and $K_{T}$ data at high temperature. Finally, we discuss the Grüneisen parameter of forsterite, in both macroscopic and microscopic terms. To complement the available experimental data base needed for our purpose, we have also redetermined the heat capacity of forsterite between 800 and $1800 \mathrm{~K}$ and measured the pressure and temperature dependences of the Raman-active vibrational frequencies of forsterite.

\section{EXPERIMENTAL METHODS}

Calorimetric measurements were made with the ice calorimeter, high-temperature equipment and experimental procedures described by Richet et al. [1982], with the slight modifications reported by Richet and Bottinga [1984]. About $5-6 \mathrm{~g}$ of forsterite, contributing from 60 to $65 \%$ to the total measured heat content, were run in a Pt-Rh $15 \%$ crucible. 
Measurements under the same conditions on $\alpha-\mathrm{Al}_{2} \mathrm{O}_{3}$, the calorimetric standard, suggest instrumental inaccuracies of about 0.2 and $0.5 \%$ for the relative enthalpies and heat capacities, respectively [Richet et al., 1982].

The forsterite specimen used for calorimetric measurements, of industrial origin, was given by $O$. Jaoul (Université Paris XI). Its composition, as determined from electron-microprobe analyses with the automated CAMEBAX microprobe of the Université Paris VI is 0.11 (2) wt \% $\mathrm{Al}_{2} \mathrm{O}_{3}, 42.61$ (3) wt \% $\mathrm{SiO}_{2}, 57.36$ (4) wt \% $\mathrm{MgO}$ and 0.05 (2) wt \% CaO, total of $100.15(6)$. This compares favorably with the nominal composition, namely 42.70 and 57.30 wt $\% \mathrm{SiO}_{2}$ and $\mathrm{MgO}$, respectively. The lattice parameters $a=4.760(1), b=10.201$ (2) and $c=5.985$ (1) $\AA$ were derived from an Xray powder diffraction pattern in which the reflections of only forsterite were apparent. Hence, the molar mass used was the nominal one. Spectroscopic measurements were performed on a synthetic specimen of similar quality.

The Raman spectra were recorded with the multichannel microprobe (Microdil 28, from Dilor) of the MLRO service of the University of Nantes. The light was collected in the backscattering direction through a Leitz UTK 40 or UTK 50 objective (focal distance of 8 or $18 \mathrm{~mm}$; numerical aperture of 0.63 or 0.32 , respectively). The spectra were obtained from about 10 accumulations lasting each 20 to $40 \mathrm{~s}$, and peak positions were identified to within $\pm 1 \mathrm{~cm}^{-1}$. To record a maximum number of bands at high temperatures, small single crystals were inserted with different orientations into the heating stage of a microscope. Temperatures were measured within a few degrees with a Pt-PtRh $10 \%$ thermocouple. A diamond-anvil cell was used to compress the sample in the $200-\mu \mathrm{m}$ hole of a stainless-steel gasket to record the highpressure spectra. A 4:1 ethanol-methanol mix was used as a pressure-transmitting medium for all experiments, and pressures were measured with the ruby-fluorescence method.

\section{CALORIMETRY}

In spite of the considerable geophysical importance of forsterite, calorimetric measurements have long been scarce and the relative enthalpies of $\mathrm{Orr}$ [1953] up to $1800 \mathrm{~K}$ were the only available high-temperature data. Our experimental relative enthalpies (Table 1 ) are about $1 \%$ lower than the results of Orr, except at the highest temperatures where the difference tends to decrease. This is apparent in Figure 1 where both data sets are plotted in the form of mean heat capacities, $C_{m}=\left(H_{\mathrm{T}}-H_{273}\right) /(T-273)$.

TABLE 1. Relative Enthalpy of Forsterite

\begin{tabular}{lcc} 
& $(\mathrm{kJ} / \mathrm{mol})$ & \\
\hline Run & $T, \mathrm{~K}$ & $H_{\mathrm{T}} H_{273}$ \\
\hline BD.8 & 787.9 & 75.718 \\
BD.3 & 922.3 & 99.519 \\
BD.11 & 977.0 & 109.12 \\
BD.9 & 1087.0 & 128.41 \\
BD.4 & 1123.3 & 135.64 \\
BD.2 & 1264.2 & 160.30 \\
BD.5 & 1374.2 & 180.44 \\
BD.1 & 1615.6 & 225.85 \\
BD.7 & 1493.8 & 202.44 \\
BD.10 & 1722.1 & 246.15 \\
BD.12 & 1847.1 & 270.75 \\
\hline
\end{tabular}

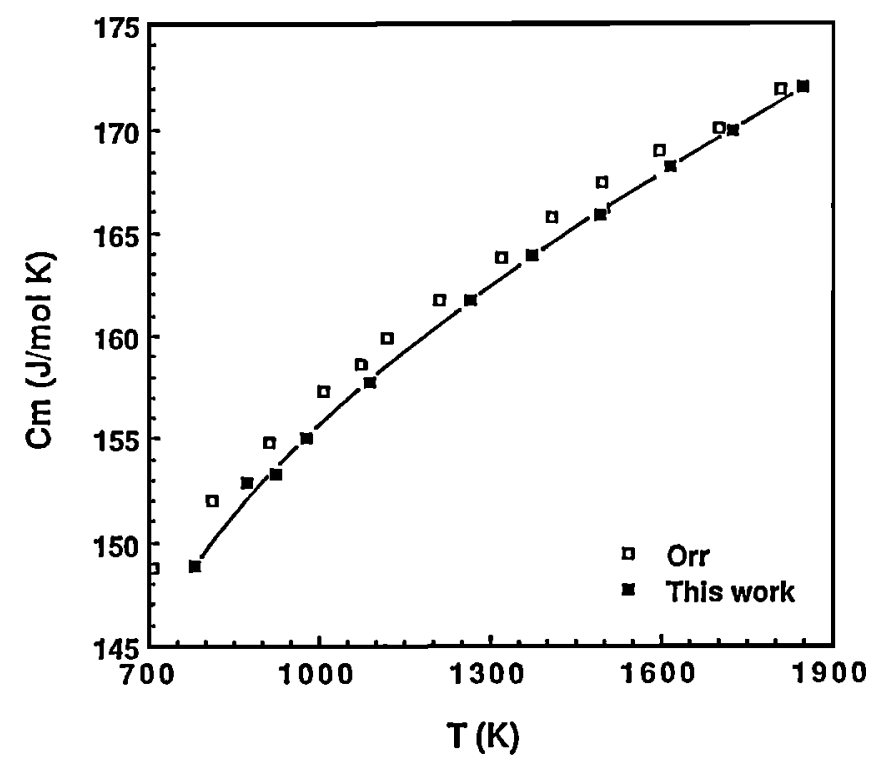

Fig. 1. Mean heat capacity of forsterite. Open squares: results of Orr [1953], referred to $273 \mathrm{~K}$ with the $\mathrm{H}_{298}-\mathrm{H}_{273}$ of Robie et al. [ 1982]; solid squares: this work.

A simultaneous least-squares fit was made to the experimental $C_{p}$ and enthalpy data to obtain the following equations of the form recommended by Haas and Fisher [1976], Berman and Brown [1985] and Richet and Fiquet [1991], respectively:

$$
\begin{aligned}
C_{p}=297.570 & -55.68410^{-3} T-5.18710^{5} / T^{2} \\
& -2731.7 T^{0.5} \\
+ & 19.79110^{-6} T^{3} \\
C_{p}=241.777+ & 2119.3 / T^{0.5}+1.565710^{6} / T^{2} \\
- & 4.725810^{8} / T^{3}, \\
C_{p}=-402.753+ & 74.290 \ln T+87.58810^{3} / T \\
& -25.91310^{6} / T^{2} \\
+ & 25.37410^{8} / T^{3}
\end{aligned}
$$

At low temperatures, the adiabatic $C_{p}$ of Kelley [1943], down to $50 \mathrm{~K}$ only, have been superseded by those of Robie et al. [1982] for the wider temperature interval 5-380 K. Our results join smoothly with both sets of measurements which are in mutual agreement, as indicated by the deviations of the values given by equations (1)-(3) from the experimental data (Table 2). These results also agree, to within their greater error margins, with the Differential Scanning Calorimetry (DSC) measurements of Watanabe [1982] and Ashida et al. [1987].

The heat capacities reported in these different studies are plotted in Figure 2. Up to $1600 \mathrm{~K}$, the consistency of all the data is to within $\pm 1 \%$ in the temperature range of the various measurements. Above $1600 \mathrm{~K}$, the anomalously high heat capacities given by the equation reported by Orr [1953] and (1) result from the analytical forms of these equations that do not allow high-temperature extrapolations. As discussed by Richet and Fiquet [1991], equation (3) is to be preferred for this purpose whereas (2) gives apparently too low values because, in contrast to (1) and (3), it does not reproduce the 
TABLE 2. Average Absolute Deviations (AAD) of Values Given by Equations (1), (2) and (3) From The Experimental $C_{p}$ and Enthalpy Data

\begin{tabular}{|c|c|c|c|c|c|c|}
\hline \multirow[b]{2}{*}{ Reference } & \multirow[b]{2}{*}{ Property } & \multirow[b]{2}{*}{$N$} & \multirow[b]{2}{*}{$\Delta T, \mathbf{K}$} & \multicolumn{3}{|c|}{ AAD\% } \\
\hline & & & & (1) & (2) & (3) \\
\hline Kelley [1943] & $c_{p}$ & 3 & $276-295$ & 0.31 & 0.39 & 0.23 \\
\hline Robie et al. [1982] & $c_{p}$ & 22 & $270-380$ & 0.08 & 0.18 & 0.06 \\
\hline Watanabe [1982] & $C_{p}$ & 8 & $350-700$ & 0.43 & 0.63 & 0.33 \\
\hline Ashida et al. [1987] & $c_{p}^{\prime}$ & 10 & $280-700$ & 1.10 & 0.75 & 0.84 \\
\hline Orr [1953] & $\dot{H}$ & 16 & $398-1808$ & 0.82 & 0.75 & 1.15 \\
\hline This Work & $\boldsymbol{H}$ & 11 & $782-1847$ & 0.05 & 0.15 & 0.06 \\
\hline
\end{tabular}

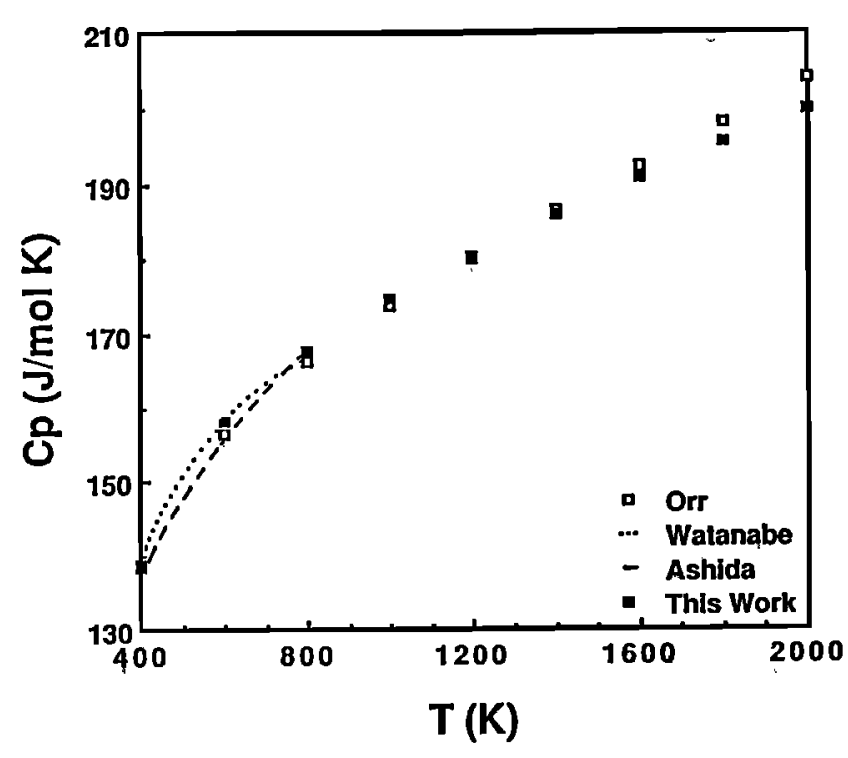

Fig. 2. Heat capacity of forsterite as reported by $\operatorname{Orr}[1953]$, Robie et al. [1982], Watanabe [1982], Ashida et al. [1987], and in this work.

experimental data to within their error margins. This is apparent in Table 2 for the measurements of Robie et al. [1982] and ours. In the following sections, the heat capacity will thus be obtained from (3), with an uncertainty that is estimated to be lower than $1 \%$ up to $2000 \mathrm{~K}$ at least.

\section{SPECTROSCOPY}

As shown below, accurate calculations of the heat capacity require comprehensive high-pressure and high-temperature spectroscopic data. Previous Raman and infrared measurements on forsterite have been reviewed by Hofmeister [1987] and McMillan and Hofmeister [1988]. Briefly, the high vibrational frequencies, between 800 and $1000 \mathrm{~cm}^{-1}$, have been assigned to combinations of symmetric and asymmetric stretching modes of the $\mathrm{SiO}_{4}$ tetrahedra. A gap in the range 800 $650 \mathrm{~cm}^{-1}$ separates these modes from the lower-frequency part of the spectrum, which includes the bending modes of the $\mathrm{SiO}_{4}$ tetrahedra and the lattice modes involving the $\mathrm{MgO}_{6}$ octahedra.

To fill in the lack of high-temperature measurements, we have recorded the Raman spectrum of forsterite up to $1150 \mathrm{~K}$ at ambient pressure. The usual band broadening at high- temperatures is apparent in Figure 3 and the negative variations with temperature of the frequencies are linear within experimental uncertainties (Table 3 and Figure 4). The relative frequency shifts plotted in Figure 5 as a function of wavenumber show that the internal modes depend less on temperature than the lattice modes. This is also apparent in Table 3 which lists the corresponding isobaric Grüneisen parameters:

$$
\gamma_{i P}=-1 / \alpha\left(\partial \ln v_{i} / \partial T\right) P
$$

which were calculated with the 1 -bar, $298 \mathrm{~K}$ thermal expansion coefficient.

At room temperature, infrared measurements up to $42.5 \mathrm{GPa}$ have been reported by Hofmeister et al. [1989], and the available Raman data [Besson et al., 1982; Gillet et al., 1988; Chopelas, 1990] extend up to $22.7 \mathrm{GPa}$. For intercomparison purposes and for estimating better the uncertainties on the shifts, we have also recorded the roomtemperature Raman spectrum of forsterite up to $5.5 \mathrm{GPa}$. Measurements were made with both increasing and decreasing pressures or temperatures. No hysteresis was observed. Typical examples of the spectra at pressure are given in Figure 6. Qualitatively, the two frequency ranges found under ambient conditions are still observed at pressure, with only linear increases of the vibrational frequencies with pressure (Table 3).

Above $10 \mathrm{GPa}$, Chopelas [1990] and Hofmeister et al. [1989] have interpreted minor changes in the vibrational pattern in terms of a phase transition and we will thus restrict ourselves to pressures lower than $10 \mathrm{GPa}$ where our highpressure results agree with the more extensive data of Chopelas [1990], see Table 3 and Figure 7. The relative changes in frequency of the internal modes of the $\mathrm{SiO}_{4}$ tetrahedra depend clearly less on pressure than those of the lattice modes, as previously pointed out by Hofmeister et al. [1989] and Chopelas [1990]. This is also shown in Table 3 by the corresporiding isothermal Grüneisen parameters:

$$
\gamma_{i T}=K_{T}\left(\partial \ln v_{i} / \partial P\right) T
$$

which were calculated with the 1-bar, $298 \mathrm{~K}$ bulk modulus.

\section{ANHARMONICITY AND HEAT CAPACITY}

\section{Macroscopic Evidence}

The isochoric heat capacity $\left(C_{v}\right)$ is related to the measurable ișobaric heat capacity by: 


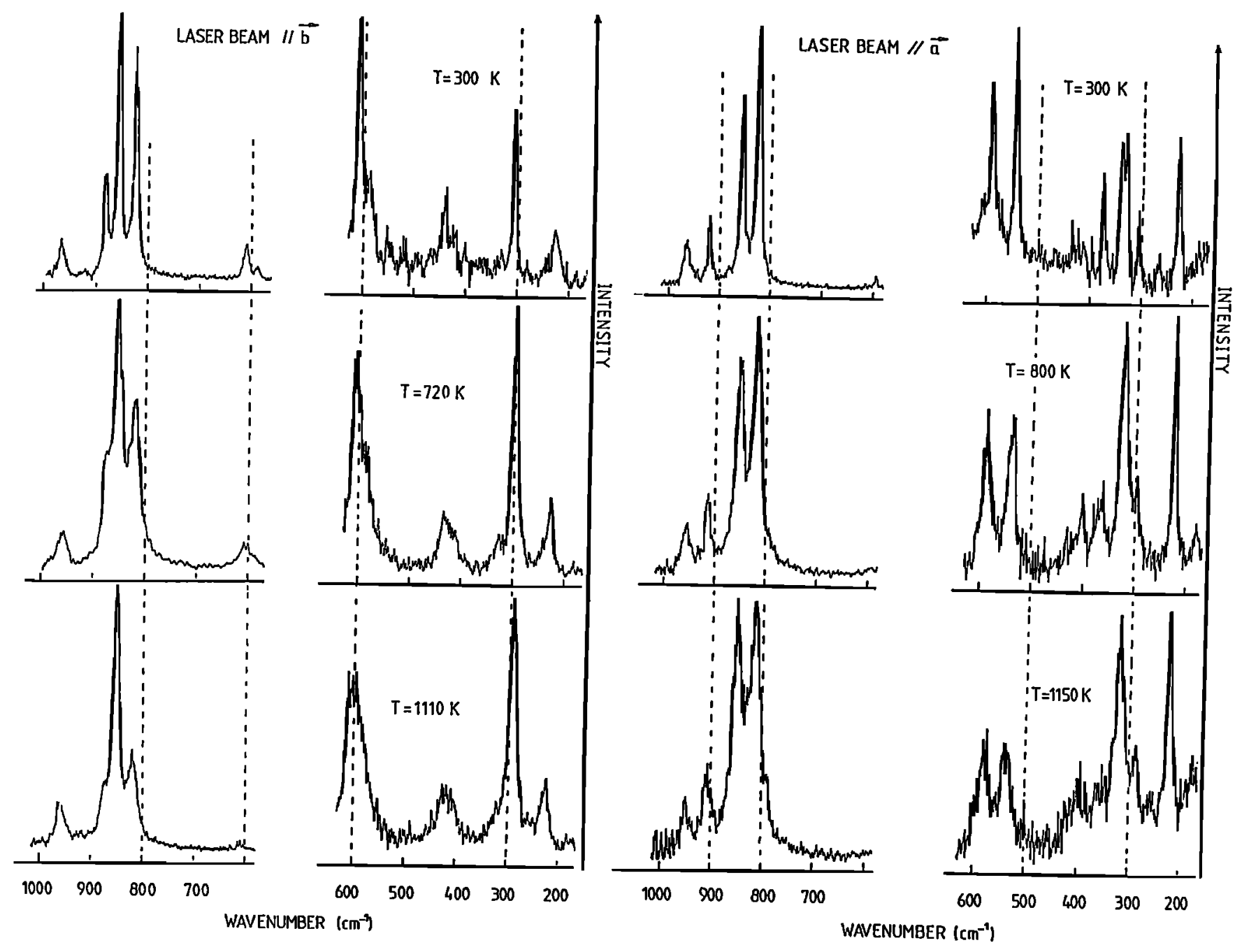

Fig. 3. Raman spectrum of forsterite at three temperatures with the laser beam parallel to either the $a$ or the $b$ axis. The intensity differences result from polarization effects inherent to the collecting geometry of the microRaman setup.

$$
C_{v}=C_{p}-T V \alpha^{2} K_{T}
$$

Departure of $C_{v}$ from the limit of Dulong and Petit, namely $3 \mathrm{R} / \mathrm{g}$ atom $(R=$ gas constant $)$ is a traditional measure of intrinsic anharmonicity. In Figure 8 we have plotted $C_{v}$ as calculated from equations (5) and (3) and the most recent hightemperature measurements of $\alpha$ and $K_{T}$ by Kajiyoshi [1986] and Isaak et al. [1989]. The Dulong-and-Petit limit is passed at about $1300 \mathrm{~K}$ and the excess $C_{v}$ over this limit reaches about 5 $\%$ at $2000 \mathrm{~K}$. In other words, the $T V \alpha^{2} K_{T}$ term of $C_{p}$ accounts for only part of the vibrational anharmonicity. As stated previously by Anderson and Suzuki [1983] from older data, forsterite thus clearly displays intrinsic anharmonicity at high temperature, a conclusion also reached when one uses other values of $\alpha$ such as those plotted in Figure 2 of Isaak et al. [1989]. Such an effect is not apparent in simple compounds like $\mathrm{MgO}$ and $\mathrm{CaO}$ for which the Dulong and Petit limit for $C_{\nu}$ is not exceeded at high temperature [Anderson and Zou, 1990]. An explanation is that in these oxydes the phonons are only weakly coupled whereas, in complex structures like forsterite, strong coupling (and thus intrinsic anharmonicity) is expected.

\section{Anharmonic Calculation of the Heat Capacity}

Heat capacity calculations are usually made within the harmonic approximation, i.e., with the assumption that vibrational frequencies are independent of temperature. Regardless of the details of this kind of analysis, the main point is that the contribution of each acoustic or optic mode to the heat capacity is in fact that of an harmonic, Einstein oscillator:

$$
C_{v i}^{h}=k\left(h v_{i} / k T\right)^{2} \exp \left(h v_{i} / k T\right) /\left[\exp \left(h v_{i} / k T\right)-1\right]^{2}
$$

where the frequency $v_{i}$ is independent of temperature, and $h$ and $k$ are the Planck and Boltzmann constants.

As shown in Figure 4 for forsterite, one observes that vibrational frequencies do vary with temperature. In the quasiharmonic approximation, these variations are simply accounted for by allowing the frequencies to be temperature dependent in equation (6). Thus, in both the harmonic and quasi-harmonic approximations, the high-temperature limit of $C_{v}$ is $3 R / \mathrm{g}$ atom since the high-temperature limit of an Einstein oscillator is $k$. In other words, it is assumed that the anharmonic contribution to $C_{v}$ is negligible, and that 
TABLE 3. Pressure and Temperature Shifts of Vibrational Frequencies, and Anharmonic Parameters

\begin{tabular}{|c|c|c|c|c|c|c|}
\hline$v_{i}, \mathrm{~cm}^{-1}$ & $\gamma T^{*}$ & $\gamma T^{\dagger}$ & $\gamma T^{\neq}$ & $\left(\partial v_{i} / \partial T\right)^{\dagger}, \mathrm{cm}^{-1} / \mathrm{K}$ & $\gamma_{p}{ }^{\dagger}$ & $\begin{array}{c}a_{i}^{\dagger} \\
10^{-5} \mathrm{~K}^{-1}\end{array}$ \\
\hline $\begin{array}{l}967 \\
920 \\
882 \\
856 \\
825 \\
610 \\
593 \\
585 \\
548 \\
443 \\
426 \\
414 \\
376 \\
341 \\
334 \\
307 \\
244 \\
232 \\
183 \\
171^{8} \\
998\end{array}$ & $\begin{array}{l}0.66 \\
0.38 \\
0.44 \\
0.49 \\
0.48 \\
0.70 \\
\\
0.66 \\
0.53 \\
1.60 \\
1.41 \\
0.99 \\
1.25 \\
1.87 \\
1.16 \\
1.63 \\
1.21 \\
0.67 \\
2.09 \\
1.47 \\
1.04\end{array}$ & $\begin{array}{l}0.7 \\
0.4 \\
0.5 \\
0.5 \\
0.5 \\
0.7 \\
\\
0.6 \\
0.6 \\
1.8 \\
\\
1.4 \\
1.3 \\
1.8 \\
0.7\end{array}$ & $\begin{array}{l}0.54 \\
0.49 \\
0.54\end{array}$ & $\begin{array}{c}-0.027(2) \\
-0.019(2) \\
-0.023(2) \\
-0.016(1) \\
-0.017(1) \\
-0.013(1) \\
-0.012(1) \\
-0.013(1) \\
-0.011(1) \\
-0.029(2) \\
-0.018(2) \\
-0.022(2) \\
-0.019(2) \\
-0.025(2) \\
-0.019(2) \\
-0.023(2) \\
-0.018(1) \\
-0.011(1) \\
-0.012(1) \\
-0.012(2) \\
-0.008(2)\end{array}$ & $\begin{array}{l}1.07(16) \\
0.82(14) \\
1.00(16) \\
0.72(10) \\
0.79(10) \\
0.82(15) \\
0.85(13) \\
0.85(13) \\
0.77(13) \\
2.52(36) \\
1.62(30) \\
2.04(30) \\
1.94(35) \\
2.82(43) \\
2.18(39) \\
2.87(46) \\
2.13(27) \\
1.82(27) \\
2.51(37) \\
2.60(62) \\
2.99(97)\end{array}$ & $\begin{array}{l}-1.07(32) \\
-1.16(35) \\
-1.46(44) \\
-0.60(18) \\
-0.81(20) \\
-0.31(9) \\
\\
-0.51(15) \\
-0.63(19) \\
-2.38(71) \\
-0.58(17) \\
-2.74(82) \\
-1.79(54) \\
-2.51(75) \\
-0.81(24) \\
-3.22(96) \\
-3.10(93) \\
-3.00(90) \\
-1.12(34) \\
-3.0(15) \\
-5.0(25)\end{array}$ \\
\hline $\begin{array}{l}\text { * Data fro } \\
+ \text { Results } \\
\text { uncertainty } \\
\neq \text { Data fro } \\
\& \text { Acousti } \\
\text { [1989]. }\end{array}$ & $\begin{array}{l}\text { Chop } \\
\text { om th } \\
\text { YiT } \\
\text { Bess } \\
\text { mode }\end{array}$ & $r$ & 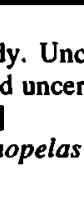 & $s$ on the $\gamma P$ & $\alpha=2.7$ & $\begin{array}{l}\mathrm{K}^{-1} \text {, a } 15 \% \\
\text { Isaak et al. }\end{array}$ \\
\hline
\end{tabular}
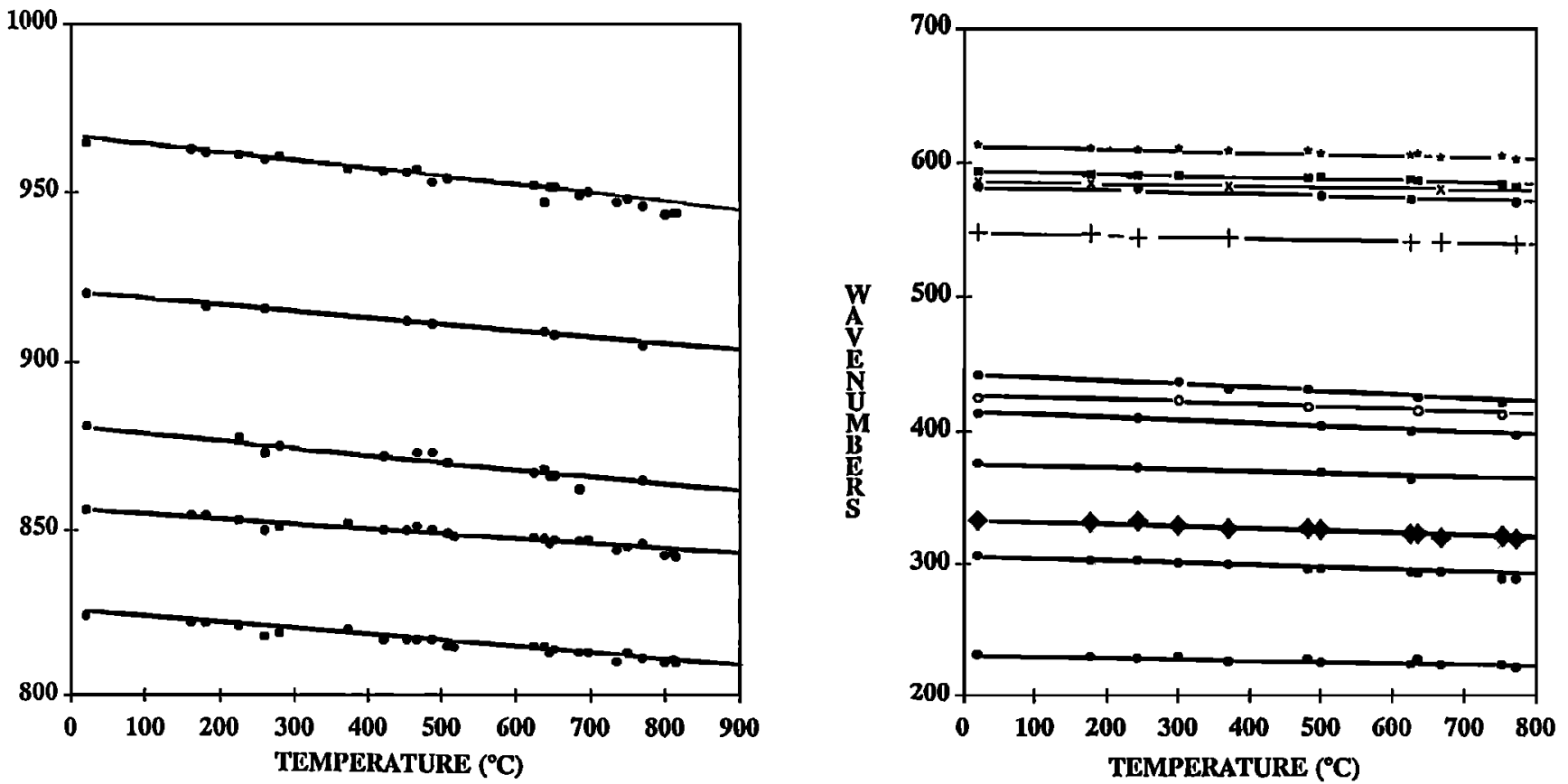

Fig. 4. Temperature dependence of the vibrational frequencies. The symbol size is similar to the errors of the data.

anharmonicity (often called extrinsic anharmonicity) contributes to $C_{p}$ in (5) through the thermal expansion coefficient $\alpha$ only.

For forsterite, Kieffer modeling [Hofmeister, 1987; Chopelas; 1990] or lattice dynamical calculations [Price et al.,
1987; Rao et al., 1988; Choudury et al,, 1989] have been performed up to $1000 \mathrm{~K}$ with either the harmonic or quasiharmonic assumptions. In view of the excess $C_{v}$ over the Dulong-and-Petit limit shown by forsterite, a calculation of the intrinsic anharmonic contribution to $C_{\nu}$ itself must be 


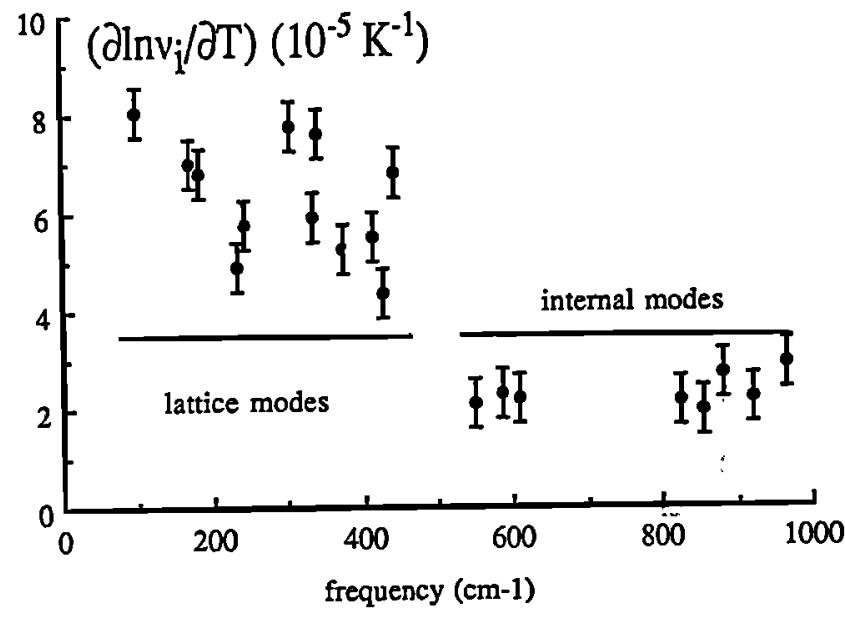

Fig. 5. Relative changes in internal and lattice vibrational frequencies with temperature. The absolute values are represented, but all the shifts are in fact negative.

performed. Gillet et al. [1989] pointed out that this contribution can be determined simply from high-pressure and high-temperature spectroscopic measurements. To demonstrate this result in a more rigorous way, we will characterize the intrinsic anharmonicity of a vibrational mode $i$ by the variation of $v_{i}$ with temperature at constant volume. We will use for this purpose the anharmonic parameters $a_{i}$ introduced by Mammone and Sharma [1979]:

$$
a_{i}=\left(\partial \ln v_{i} / \partial T\right) V=\alpha K_{T}\left(\partial \ln v_{i} / \partial P\right) T+\left(\partial \ln v_{i} / \partial T\right) P
$$

The parameter $a_{i}$ can also be expressed as

$$
a_{i}=\alpha\left(\gamma_{i}-\gamma_{i P}\right)
$$

and thus it can be determined from the pressure and temperature dependences of $v_{i}$ through $(4 a)-(4 b)$. Theșe parameters at $298 \mathrm{~K}$ and $1 \mathrm{bar}$ are listed in Table 3 for forsterite, along with their uncertainties as obtained from the spectroscopic results. Note in Figure 9 that the absolute values of these parameters are lower for the internal mades of $\mathrm{SiO}_{4}$ tetrahedra than for the lattice modes.

As we will show now, the usefulness of the $a_{i}$ parameters is that the isochoric heat capacity of an ensemble of anharmonic oscillators is simply related to the harmonic heat capacities:

$$
C_{v}=\sum C_{v i}^{h}\left(1-2 a_{i} T\right)=C_{v}^{h}-T \sum 2 a_{i} C_{v i}^{h}
$$

where $C_{v}{ }^{h}$, the harmonic part of the heat capacity, can be obtained with a suitable model in which the room-temperature and pressure frequencies are used. To demonstrate equation (9), we begin with the internal energy of the ensemble of oscillators which is related to the partition functions of the individual oscillators $\left(Z_{i}\right)$ by:

$$
U=U_{0}+\Sigma k T^{2}\left(\partial \ln Z_{i} / \partial T\right) V
$$

where $U_{0}$ is the lattice cohesive energy. For an harmonic oscillator, the partition function is:

$$
Z_{i}=\exp \left(-h v_{i} / 2 k T\right) /\left[1-\exp \left(-h v_{i} / k T\right)\right]
$$
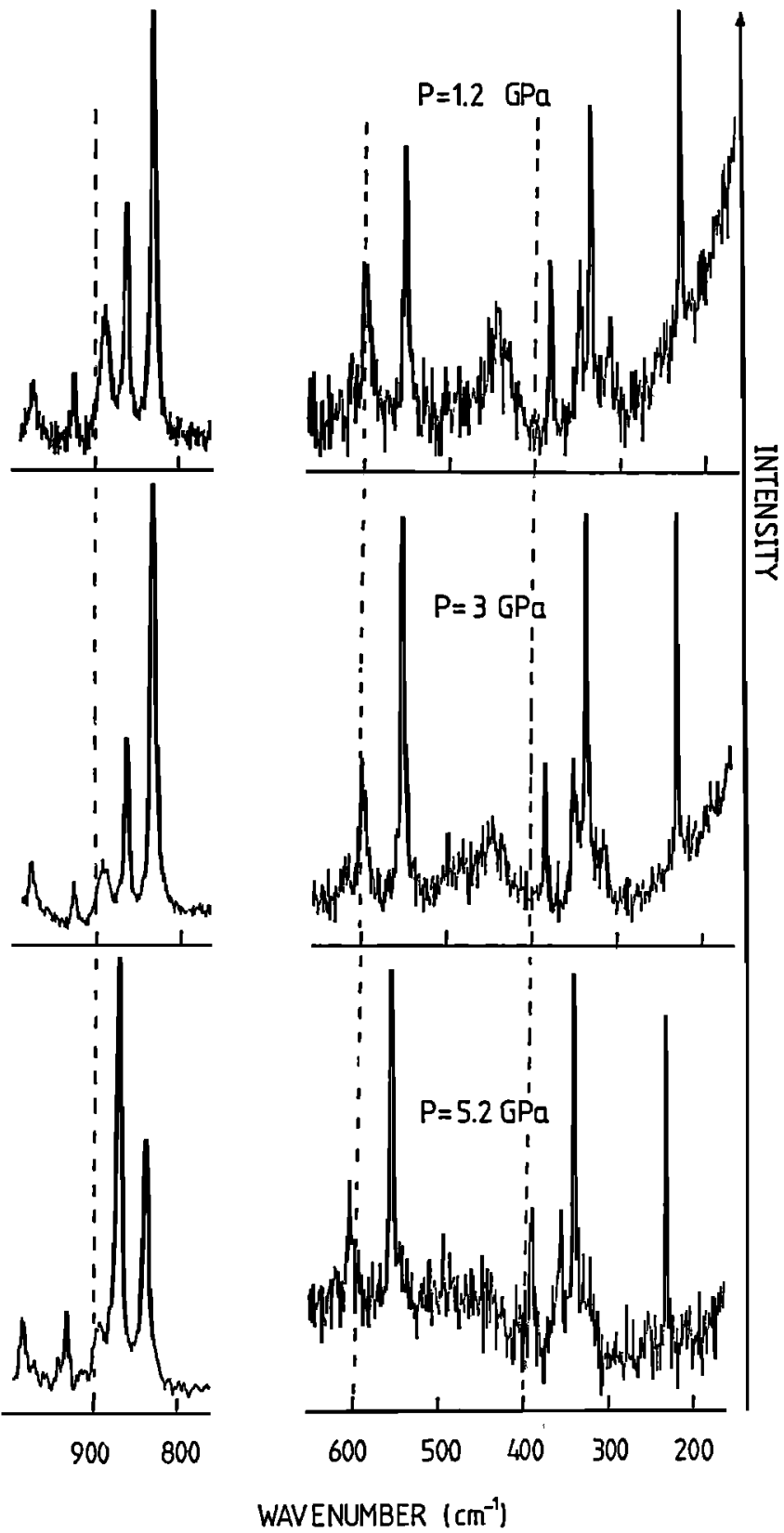

Fig. 6. Raman spectrum of forsterite at three pressures.

Spectroscopic measurements show that $\left(\partial \ln v_{i} / \partial T\right)_{V}$ generally differ from zero. Thus we will use (11) but we take into account the variation's of the frequencies with temperature at constant volume when inserting (11) in (10) to obtain:

$$
U=U_{0}+\sum U_{i}^{h}\left(1-a_{i} T\right)
$$

where the harmonic vibrational internal energy is

$$
U_{i}^{h}=h v_{i}\left\{1 / 2-1 /\left[1-\exp \left(h v_{i} / k T\right)\right]\right\}
$$

The anharmonic $C_{v}$ is thus

$$
\begin{gathered}
C_{v}=(\partial U / \partial T) V \\
=\Sigma\left(C_{v i}^{h}\left(1-a_{i} T\right)^{2}-T U_{i}^{h}\left[a_{i}^{2}+\left(\partial a_{i} / \partial T\right) V\right]\right\}
\end{gathered}
$$




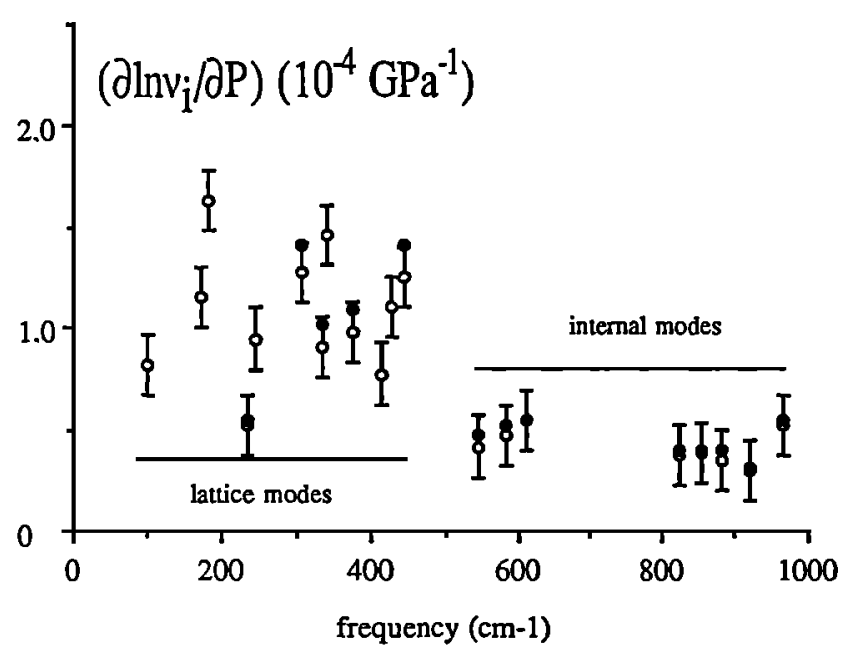

Fig. 7. Relative changes in internal and lattice vibrational frequencies with pressure. Open circles: Chopelas [1990]; solid circles: this work.

In the next section, we evaluate the various terms of (14) to show that this expression reduces numerically to the much simpler equation (9). Gillet et al. [1989] obtained previously for $C_{\nu}$ :

$$
C_{v}=\sum C_{v i}^{h}-\sum a_{i} U_{i}^{h}-T \sum a_{i} C_{v i}^{h}
$$

Equation (14) is more rigorous than (15) because it incorporates the contribution of the zero-point energy and takes consistently into account the variation with temperature of the vibrational frequencies. Numerically, however, (15) also reduces to (9) because $U_{i}^{h}$ do not differ much from $T C_{v i}{ }^{h}$ at high temperatures. For practical purposes, (9), (14) and (15) thus give nearly the same results.

\section{Numerical Calculations}

From the spectroscopic data, one finds that all the $a_{i}$ parameters are of the order of $-10^{-5} \mathrm{~K}^{-1}$ (cf. Table 3). Hence, the first term of the right-hand side of (14) can be safely

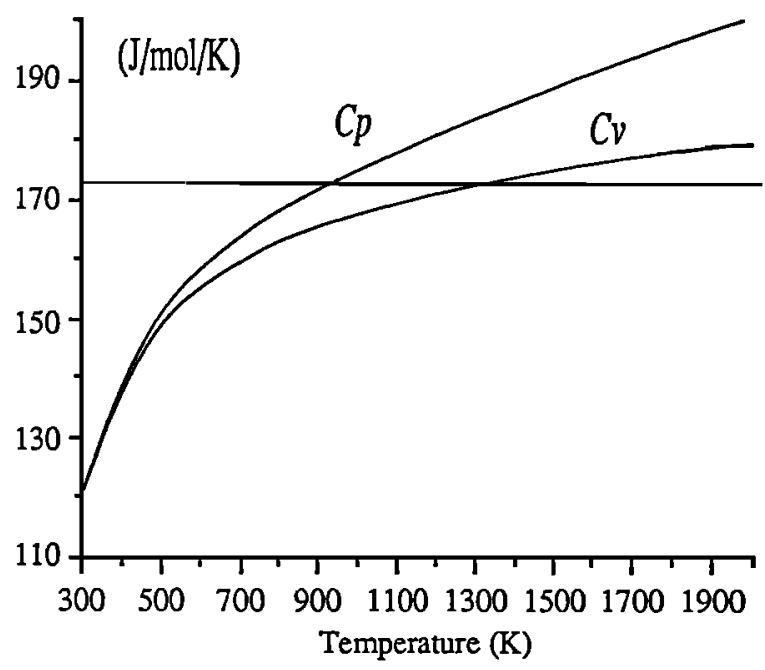

Fig. 8. Isobaric and isochoric heat capacity of forserite: $C_{p}$ : given by equation (3), and $C_{\nu}$ calculated from relation (5) with $\alpha$ and $K_{T}$ as given by Isaak et al. [1989].

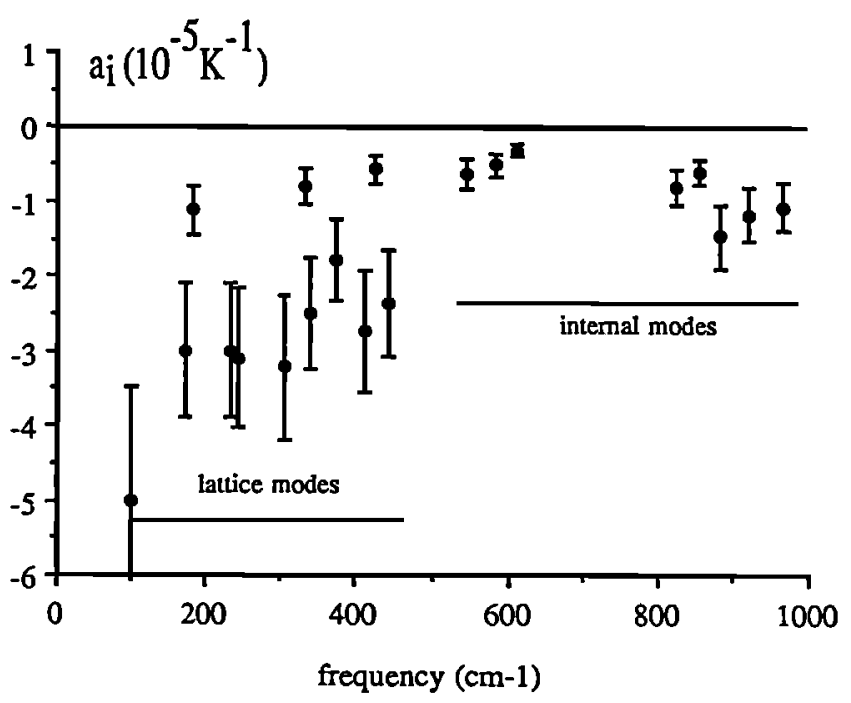

Fig. 9. Anharmonic parameters of the Raman modes of forsterite calculated at $298 \mathrm{~K}$ and 1 bar.

reduced to (9) up to a few thousand degrees. To show that the second term is negligible, we note that the term $T U_{i}{ }^{h}$ is about $T^{2} C_{v i}{ }^{h}$ at high temperatures. Finally, $\left(\partial a_{i} / \partial T\right) V$ can be determined from

$$
\left(\partial a_{i} / \partial T\right)_{V}=\left(\partial a_{i} / \partial T\right)_{P}+\alpha K_{T}\left(\partial a_{i} / \partial P\right) T
$$

From our results which show linear variations of the frequencies with pressure and temperature, we estimate that the two derivatives of the RHS of (16) are smaller than $10^{-9} \mathrm{~K}^{-1}$. This makes it these terms negligible in (16). In summary, the parameters $a_{i}$ must be considered as temperature independent in (9), a conclusion similar to that obtained by Gillet et al. [1990] for quartz from detailed numerical calculations of the $a_{i}$ parameters.

\section{Application to Forsterite}

All optic vibrational modes are not Raman or infrared active. For practical applications, it is thus necessary to use an averaging scheme for the observed frequencies. In Kieffer's model the contribution to $C_{v}$ of a set of $m$ optic continua is given by

$$
\begin{gathered}
C_{v}=3 n R \sum_{i=1}^{m} \frac{n_{i}}{N} \int_{v_{l i}}^{v_{u i}} \frac{x^{2} \exp (x) d x}{\left(v_{u i}-v_{l i}\right)\left[\exp (x)-1^{2}\right]} \\
=3 n R \sum_{i=1}^{m} C_{v i}{ }^{h}
\end{gathered}
$$

with $x=h v_{i j} k T, n$ is the number of atoms in the mineral formula, $R$ is the gas constant, $n_{i}$ is the number of modes in the ith continuum, $N$ is the total number of vibrational modes, and $v_{i}$ and $v_{u i}$ are the lower and upper cutoff frequencies of the ${ }_{i}$ th continuum, respectively.

Using Kieffer's [1979] model with several optic continua, Hofmeister [1987] has proposed different approximations for the density of states of the optical modes of forsterite. Two of these models are shown in Figure 10, along with a third one which we have set up to account for the three different ranges of 

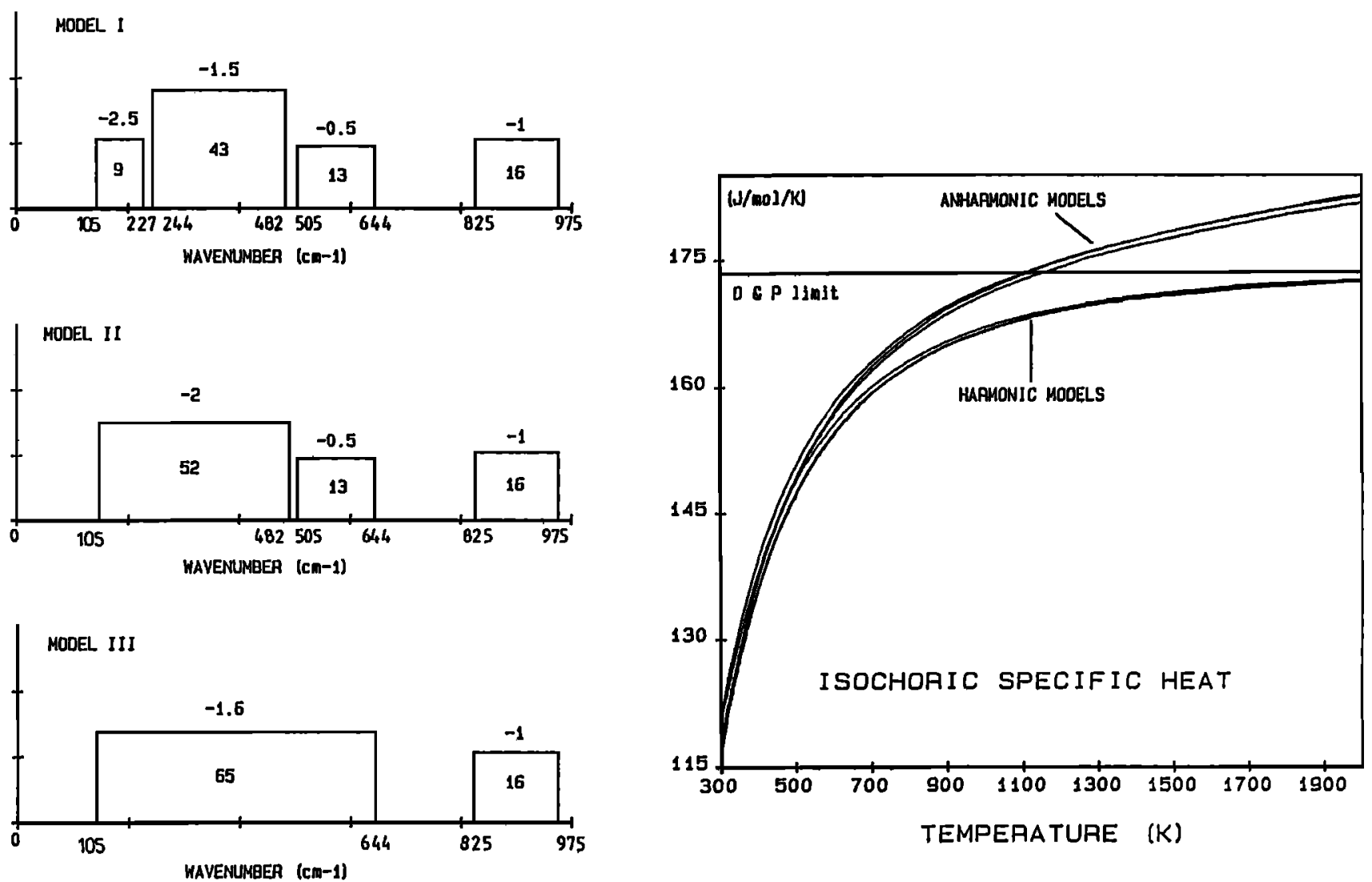

a

b

Fig. 10. (a) Models of density of states consistent with the spectroscopic data (acoustic modes not shown). Models I and III are adapted from Hofmeister [1987]. The lowest mode at $105 \mathrm{~cm}^{-1}$ is taken from the inelastic neutron scattering data of Rao et al. [1988]. The numbers in the boxes represent the number of modes in that continuum. The numbers above the boxes refer to the mean $a_{i}$ parameter for those modes. (b) Harmonic and anharmonic $C_{v}$ calculated with the densities of states shown in (a).

frequencies apparent in Figures 5 and 7. The numerical data relevant to heat capacity calculations with these three models are included in Figure 10. The remaining data, namely the acoustic velocities and molar volumes, were taken from Kieffer [1979] and Hofmeister [1987]. As usual, the results are sensitive functions of the cutoff frequencies at low temperatures, but the main result is that all models yield harmonic heat capacities differing by less than 2 and $0.5 \%$ at 300 and $1000 \mathrm{~K}$, respectively (Figure 10 and Table 4).

With the anharmonic contribution, the isochoric heat capacity is

$$
C_{v}=3 n R \sum_{i=1}^{m} C_{v i}{ }^{h}\left(1-2 a_{i} T\right)
$$

For evaluating (18) an averaging scheme must also be used for the $a_{i}$ parameters. To ensure consistency with the calculation of the harmonic contribution to $C_{\nu}$, the $a_{i}$ were averaged over the same frequency continua as used in the various Kieffer models. Calculations show that the anharmonic contribution becomes significant at about $1300 \mathrm{~K}$ (Figure 10 and Table 4) and reaches about $7 \%$ at $2000 \mathrm{~K}$, with values that are insensitive to the averaging scheme, and brings the calculated $C_{y}$ to within $1 \%$ with the values obtained from measurements with equation (5).

\section{CONSISTENCY OF THERMODYNAMIC DATA}

The data plotted in Figure 11 illustrate the aforementioned uncertainties on the thermal expansion coefficient of forsterite. Published values differ by $30 \%$ between 300 and $1400 \mathrm{~K}$ and the differences reach $50 \%$ when extrapolating these results up to $2000 \mathrm{~K}$. To improve thermodynamic modeling, Fei and Saxena [1987a] and Saxena [1988, 1989] pointed out that equation (5) provides a simple means of ensuring consistency between thermal and volume properties. For $\mathrm{MgO}$, for example, Saxena [1989] used experimental $C_{p}$ values to optimize simultaneously $\alpha, K_{T}$ and $C_{v}$. (In passing, we note that satisfactory results could be obtained only when $C_{v}$ was allowed to differ from the Dulong-and-Petit limit, i.e., when significant anharmonicity was assumed.)

Basically, we will follow the same approach, with the main difference that we greatly reduce the number of adjustable parameters by considering $C_{v}$ as a known quantity obtained from anharmonic vibrational modeling. The optimization method followed has been described in detail by Tarantola and Valette [1982] and Sotin [1986]. The starting parameters are listed in Table 5, along with all the estimated uncertainties which are the greatest for the thermal expansion coefficient. Various analytical expressions have been used for $\alpha$ and $K_{T}$. The results depend little on the form of these equations and the following simple expressions have been found satisfactory: 
TABLE 4. Isobaric and isochoric heat capacity of forsterite ( $/ \mathrm{mol} \mathrm{K})$.

\begin{tabular}{|c|c|c|c|c|c|c|c|c|}
\hline \multirow[t]{2}{*}{$T(\mathrm{~K})$} & \multirow{2}{*}{$\begin{array}{c}C_{p} \\
\exp ^{*}\end{array}$} & \multirow{2}{*}{$\begin{array}{c}c_{v} \\
\exp ^{\dagger}\end{array}$} & \multicolumn{6}{|c|}{ calculated $C_{v}$ with models $\neq$} \\
\hline & & & $1 \mathrm{~h}$ & 1 anh & $2 \mathrm{~h}$ & 2 anh & $3 \mathrm{~h}$ & 3 anh \\
\hline $\begin{array}{l}300 \\
600 \\
900 \\
1200 \\
1500 \\
1800 \\
2000\end{array}$ & $\begin{array}{l}119.2 \\
158.2 \\
171.4 \\
180.4 \\
188.2 \\
195.7 \\
199.6\end{array}$ & $\begin{array}{l}117.9 \\
154.7 \\
165.6 \\
170.6 \\
175.0 \\
178.4 \\
180.0\end{array}$ & $\begin{array}{l}116.8 \\
154.4 \\
164.9 \\
168.9 \\
170.9 \\
172.1 \\
172.3\end{array}$ & $\begin{array}{l}117.7 \\
156.9 \\
168.9 \\
172.8 \\
178.4 \\
180.8 \\
181.2\end{array}$ & $\begin{array}{l}120.1 \\
155.4 \\
165.3 \\
169.2 \\
171.1 \\
172.1 \\
172.3\end{array}$ & $\begin{array}{l}121.1 \\
158.2 \\
169.8 \\
175.4 \\
178.4 \\
181.5 \\
182.1\end{array}$ & $\begin{array}{l}116.7 \\
154.2 \\
164.8 \\
168.9 \\
170.9 \\
172.1 \\
172.3\end{array}$ & $\begin{array}{l}117.5 \\
156.9 \\
169.1 \\
174.7 \\
177.6 \\
180.9 \\
181.6\end{array}$ \\
\hline
\end{tabular}

* Values obtained with equation (3).

$\dagger$ Calculated from equations (3) and (5), the bulk moduli of Isaak et al. [1989] and the thermal expansion of Kajiyoshi [1986].

₹ Calculated from harmonic (h) or anharmonic (anh) vibrational modeling. The numbers refer to the various densities of states of Figure 10.

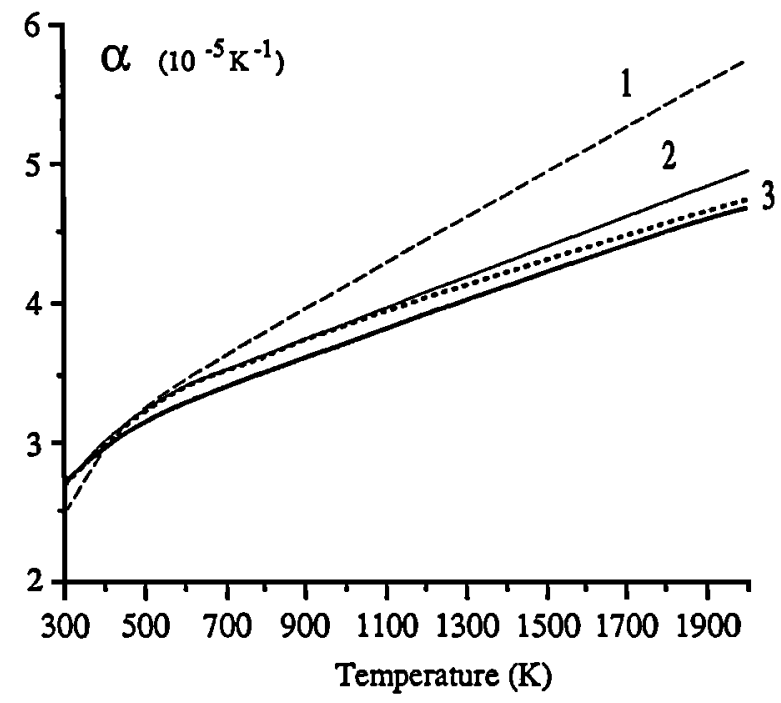

Fig. 11. Thermal expansion coefficient of forsterite. Curve 1 (dashed line) from Suzuki et al. [1983]; Curve 2 (thin line) from Kajiyoshi [1986]; curve 3 (dotted line) from Fei and Saxena [1987b]; Thick curve obtained in this work from data inversion with (5).

$$
\begin{gathered}
\alpha=\alpha_{0}+\alpha_{1} \mathrm{~T}+\alpha_{2} / T^{2} \\
K_{T}=K_{T 0}+\left(\mathrm{d} K_{T} / \mathrm{d} T\right)_{0} T .
\end{gathered}
$$

Finally, the slight temperature dependence of the volume, which plays only a minor role in (5), was accounted for by

$$
V=V_{0}\left(1+\int \alpha \mathrm{d} T\right)
$$

In summary, numerical values of $C_{p}$ taken at $50 \mathrm{~K}$ intervals as given by equation (3) were used in (5), along with the anharmonic $C_{v}$ calculated with the model \#3 of Figure 10 , to adjust the coefficients of (19) and (20).

The slight curvature with temperature of the adiabatic bulk modulus $\left(K_{s}\right)$ observed by Isaak et al. [1989] cannot be translated to the isothermal $K_{T}$ in view of the stated uncertainties of the other data appearing in equation (5).

\begin{tabular}{|c|c|c|c|c|}
\hline \multirow[b]{2}{*}{$T, \mathrm{~K}$} & \multicolumn{2}{|c|}{$\begin{array}{c}\text { Input Data } \\
\text { (1\% Uncertainty) }\end{array}$} & \multicolumn{2}{|c|}{$\begin{array}{l}\text { Inverted Values } \\
\text { and Uncertainties }\end{array}$} \\
\hline & $c_{p}$ & $C_{v}$ & $c_{p}$ & $c_{v}$ \\
\hline $\begin{array}{l}300 \\
600 \\
900 \\
1200 \\
1500 \\
1800 \\
2000\end{array}$ & $\begin{array}{l}119.2 \\
158.2 \\
171.4 \\
180.4 \\
188.2 \\
195.2 \\
199.6\end{array}$ & $\begin{array}{l}117.8 \\
156.9 \\
168.8 \\
172.8 \\
177.6 \\
180.8 \\
181.2\end{array}$ & $\begin{array}{l}117.9 \\
158.7 \\
172.7 \\
181.3 \\
188.6 \\
196.2 \\
201.7\end{array}$ & $\begin{array}{l}116.7 \\
155.4 \\
167.2 \\
172.8 \\
176.8 \\
179.2 \\
182.1\end{array}$ \\
\hline \multicolumn{2}{|c|}{ Parameters ${ }^{\dagger}$} & $\begin{array}{l}\text { Apriori Values and } \\
\text { Uncertainties }\end{array}$ & \multicolumn{2}{|c|}{$\begin{array}{l}\text { Aposteriori Values and } \\
\text { Uncertainties }\end{array}$} \\
\hline \multicolumn{2}{|c|}{$\begin{array}{l}10^{5} \alpha_{0} \\
10^{8} \alpha_{1} \\
\alpha_{2} \\
K_{T}(\mathrm{kbar}) \\
\left(d K_{T} / d T\right)(\mathrm{kbar} / \mathrm{K}) \\
V_{o}\left(\mathrm{~cm}^{3} / \mathrm{mol}\right)\end{array}$} & $\begin{array}{c}3(2) \\
1(2) \\
1(10) \\
1290(20) \\
-0.23(20) \\
43.67(30)\end{array}$ & \multicolumn{2}{|c|}{$\begin{array}{c}2.77(9) \\
0.97(9) \\
-0.32(54) \\
1277(20) \\
-0.20(2) \\
43.55(41)\end{array}$} \\
\hline
\end{tabular}

TABLE 5. Thermal Expansion and Bulk Modulus of Forsterite up to $2000 \mathrm{~K}$ From Data Inversion

"Input data for $C_{p}$ as given by equation (3), and $C_{\nu}$ (anharmonic vibrational model 3 of Figure 10 ) taken every $50 \mathrm{~K}$ from 300 to 2000 K.

$\dagger$ Initial estimates of $\alpha, K_{T}$, and $\left(d K_{T} / d T\right)$ taken from Fei and Saxena [1987b] and Isaak et al. [1989], respectively.

Comparison of the derived $\alpha$ (Table 5) with available data in Figure 11 shows very good agreement with the hightemperature observations and extrapolations of Kajiyoshi [1986]. The inverted values of $K_{T 0}$ and $\left(\mathrm{d} K_{T} / \mathrm{d} T\right)_{0}$ listed in Table 5 are also in excellent agreement with those of Isaak et al. [1989].

Finally, the consistency of our derived data can be checked independently by calculating $K_{T}$ from the measured $K_{S}$ of Isaak et al. [1989], our measured $C_{p}$, and our calculated anharmonic $C_{v}$ with:

$$
K_{T}=K_{s} C_{v} / C_{p}
$$

The moduli obtained in this way are compared in Table 6 and 
TABLE 6. Bulk modulus of forsterite (GPa)

\begin{tabular}{rrrrr}
\hline$T, K$ & $K_{S}{ }^{*}$ & $K_{T}{ }^{\dagger}$ & $K_{T}{ }^{\star}$ & $K_{T}{ }^{8}$ \\
\hline 300 & 128.7 & 127.1 & 127.7 & 127.4 \\
600 & 123.8 & 122.7 & 121.7 & 120.9 \\
900 & 118.4 & 116.8 & 115.7 & 114.1 \\
1200 & 113.0 & 109.4 & 109.7 & 107.0 \\
1500 & 107.6 & 101.5 & 103.7 & 100.0 \\
1800 & 101.9 & 94.2 & 97.7 & 93.1 \\
2000 & 98.2 & 89.3 & 91.7 & 88.3
\end{tabular}

* Adiabatic data from Isaak et al. [1989].

$\dagger$ Calculated from $K_{T}=K_{s} C_{v} / C_{p}$.

$\neq$ Obtained from data inversion with (5).

$\S$ Isaak et al. [1989].

Figure 12 with those obtained by our inversion procedure and the experimental results of Isaak et al. [1989]. All the values agree to within $2-4 \%$ over the $300-2000 \mathrm{~K}$ range.

\section{GRUNEISEN PARAMETERS}

\section{Thermal and Microscopic Parameters}

The properties studied in the previous sections are also involved in the calculation of the thermal Grüneisen parameter, $\gamma$, which is extensively used for calculating the adiabatic gradient of the mantle. Macroscopically, $\gamma$ is defined by

$$
\gamma=\alpha K_{T} V / C_{V}=V[\partial P / \partial U]_{V}
$$

and the values calculated with our anharmonic $C_{v}$ and the $\alpha$ and $K_{T}$ values obtained from our data inversion are given in Table 7 . The results agree to within $10 \%$ with the figures reported by Isaak et al. [1989] or While et al. [1985] which are also included in Table 7.

Originally, this parameter was introduced by Grüneisen [1912] in a microscopic way in the form of a vibrational mode parameter $\gamma_{i T}$ :

$$
\gamma_{i T}=-\left(\partial \ln v_{\mathbf{i}} / \partial \ln V\right) T
$$

We will note $\gamma_{m}$ the Grüneisen parameter which can be expressed in terms of these microcopic parameters. For the special case of an harmonic solid, one has [Slater, 1939]

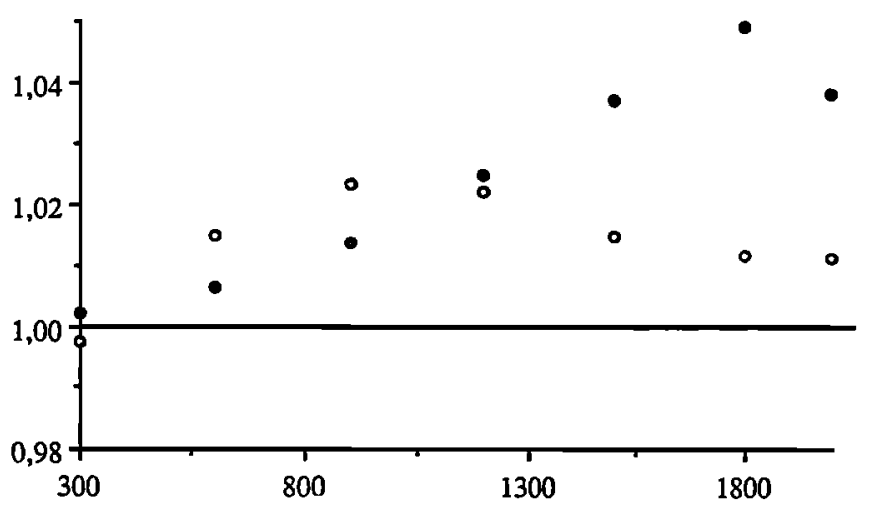

Temperature (K)

Fig. 12. Comparison of the $K_{T}$ values of Isaak et al. [1989] with those obtained from data inversion with (5) (open circles) or from (22) (solid circles). Values taken from Table 6 are nomalized to the values of $K_{T}$ of Isaak et al. [1989].

\begin{tabular}{|c|c|c|c|c|c|}
\hline \multirow[b]{2}{*}{$T, \mathrm{~K}$} & \multicolumn{2}{|c|}{ macroscopic } & \multicolumn{3}{|c|}{ microscopic } \\
\hline & $\gamma^{*}$ & $\gamma^{\star}$ & $y_{n}{ }^{\neq}$ & $\gamma_{m}{ }^{\S}$ & $\gamma_{m} \#$ \\
\hline 300 & 1.29 & 1.28 & $1.19[1.22]$ & $1.21[1.24]$ & $1.36[1.38]$ \\
\hline 600 & 1.17 & 1.10 & $1.06[1.09]$ & $1.10[1.13]$ & $1.29[1.32]$ \\
\hline 900 & 1.15 & 1.07 & $0.99[1.02]$ & $1.03[1.06]$ & $1.23[1.27]$ \\
\hline 1200 & 1.15 & 1.07 & $0.93[0.97]$ & $0.97[1.01]$ & $1.16[1.21]$ \\
\hline 1500 & 1.15 & 1.07 & $0.87[0.92]$ & $0.91[0.96]$ & $1.09[1.15]$ \\
\hline 1800 & 1.14 & 1.06 & $0.81[0.87]$ & $0.85[0.91]$ & $1.03[1.10]$ \\
\hline 2000 & 1.14 & 1.06 & $0.76[0.83]$ & $0.79[0.86]$ & $0.99[1.06]$ \\
\hline
\end{tabular}

TABLE 7. Grüneisen parameters of forsterite

* Data from Isaak et al. [1989], obtained from (23).

$\dagger$ Calculated from equation (23) with the present $\alpha, K_{T}$ and $C_{\psi}$.

$\neq$ Calculated with equation (29) and $K_{T}=128 \mathrm{GPa}$; all the modes are used. The first value is obtained with all terms of the right-hand side of (29) , the values in brackets represent the first term of the right-hand side of (29).

$\$$ Same as previous column but the $\gamma_{T T}$ of the stretching modes of the $\mathrm{SiO}_{4}$ tetrahedra are calculated with the $\mathrm{Si}-\mathrm{O}$ bulk modulus of $190 \mathrm{GPa}$ reported by Kudoh and Takéuchi [1985].

\# Calculated with equation (29) with the lattice modes only $\left(v_{i}<500\right.$ $\mathrm{cm}^{-1}$ ).

$$
\gamma_{m}=\Sigma \gamma_{i} C_{v i}^{h / \Sigma} C_{v i}^{h}
$$

where the harmonic calculation is emphasized by the superscript $h$.

The parameters calculated with (23) and (25) should be equal, but it has been found that eqn (25) gives values too small with respect to those obtained with (23) [e.g., Chopelas, 1990]. Price et al. [1987] suggested that part of the discrepancy arises from the intrinsic anharmonic contribution to $\gamma_{m}$. Hence, in this section we will first derive a general relationship between $\gamma_{m}$ and the individual mode parameters in the case of an anharmonic solid. But application of this relationship to forsterite, for which a great many mode parameters can be determined [Chopelas, 1990; this work] still underestimates $\gamma$. We will finally discuss possible reasons for the discrepancy between the macroscopic and microscopic parameters and describe a sampling method which would allow to obtain consistency between the microscopic and macroscopic thermal parameters.

\section{Anharmonic Grüneisen Parameters}

The Helmholtz free energy of an harmonic crystal is

$$
\begin{gathered}
F=F_{0}-k T \sum \ln Z_{\mathrm{i}} \\
=F_{0}+k T \sum\left\{\left(h v_{i} / 2 k T\right)+\ln \left[1-\exp \left(h v_{i} / k T\right)\right]\right\} \\
=F_{0}+k T \sum U_{\mathrm{i}}^{h} .
\end{gathered}
$$

Differentiating with respect to volume, one has

$$
P=-(\partial F / \partial N)_{T}=P_{0}+(1 / N) \Sigma \gamma_{i} U_{\mathrm{i}}^{h},
$$

where $P_{0}$ is the pressure at $0 \mathrm{~K}$. This expression is in fact the Mie-Grüneisen equation of state. Differentiating further and making use of (12), in introducing intrinsic anharmonicity one obtains

$$
\begin{aligned}
(\partial P / \partial T)_{V}= & (1 / V) \Sigma\left(\gamma_{i} T\left[a_{i} U_{\mathrm{i}}^{h}+C_{v l}{ }^{h}\left(1-a_{i} T\right)\right]\right. \\
& \left.+(\partial \gamma T / \partial T) V U_{\mathrm{i}}^{h}\right\}=\alpha K_{T} .
\end{aligned}
$$


Finally, the microscopic Grüneisen parameter is thus given by:

$$
\begin{gathered}
\gamma_{m}=\sum \gamma_{i T} C_{v i}^{h} / C_{v}+\sum \gamma_{T} a_{i}\left(U_{\mathrm{i}}^{h}-T C_{v i}^{h}\right) / C_{v} \\
+\sum \gamma_{i} T\left(\partial \gamma_{T} / \partial T\right) v U_{\mathrm{i}}^{h} / C_{v} .
\end{gathered}
$$

This equation, which accounts for anharmonicity, represents so far the most general relation between the thermal and the microscopic Grüneisen parameters. In the limit of high temperatures, this equation reduces to

$$
\gamma_{\mathrm{m}}=\Sigma \gamma_{i} T^{\prime n}+T \Sigma\left(\partial \gamma_{i T} / \partial T\right) V
$$

and we thus conclude as usual that $\gamma_{\mathrm{m}}$ is the average of the mode Grüneisen parameters only if these are not functions of temperature at constant volume.

\section{Application to Forsterite}

In this section, we assess the importance of the various terms of (29) from the spectroscopic measurements on forsterite. Only the optic modes are taken into account. Because the data for all active modes are not known, we represent the 16 modes lying between 800 and $1000 \mathrm{~cm}^{-1}$ by the five well-determined Raman modes which are treated as Einstein oscillators. The remaining 65 modes are modeled by the other 13 observed Raman modes.

The major term of the right-hand side of equation (29) is the first one. The variation of $\left(\partial v_{i} / \partial P\right) T$ with temperature is unknown because of the lack of spectroscopic measurements at simultaneously high pressure and temperature. Hence, we will first assume that this derivative is constant and evaluate (29) as a function of $T$ from the actual values of $K_{T}$ and $v_{i}$ at different temperatures. With this assumption, this term decreases from 1.22 at $300 \mathrm{~K}$ to 0.97 at $1200 \mathrm{~K}$ and 0.83 at $2000 \mathrm{~K}$ (Table 7). The two other terms of the right-hand side of (29) are negative and contribute significantly less, being of the order of $10^{-1}$ and $10^{-2}$ between 300 and $2000 \mathrm{~K}$, respectively (Table 7). Their effect is to lower the values calculated with only the first term of the right-hand side of (29) by $2-3 \%$ at $300 \mathrm{~K}$ and $10 \%$ at $2000 \mathrm{~K}$. If the agreement between thermal Grüneisen parameters calculated with equations (23) and (29) is fair at $300 \mathrm{~K}$, the parameter calculated with (23) reaches a hightemperature limit of about 1.15 [Isaak et al., 1989] or 1.06 (this work) whereas that obtained from (29) decreases continuously with temperature down to 0.8 at $2000 \mathrm{~K}$. The disagreement at high temperatures is thus real, even when considering the scatter in the reported macroscopic $\gamma$ [Boehler, 1982; Anderson and Suzuki, 1983; Isaak et al., 1989].

This difference can be attributed to several effects. First, too few modes would be taken into account in the calculation of $\gamma_{\mathrm{m}}$ with (29), i.e., the 18 Raman modes used would not represent correctly the optic modes of forsterite. This explanation appears unlikely, however, because similar results are obtained when the IR data of Hofmeister et al. [1989] for 15 additional modes are included in the calculations.

Another reason for the discrepancy could originate in the fact that forsterite has strong ( $\mathrm{Si}-\mathrm{O})$ and weak $(\mathrm{Mg}-\mathrm{O})$ bonds [Hazen and Finger, 1982; Kudoh and Takeuchi, 1985]. The existence of contrasted bonds is partly reflected in the $(\partial$ $\left.\ln v_{i} / \partial P\right)_{T}$ values which are smaller for the Si-O stretches than for the lattice modes (Figure 7). The above observations suggest, as emphasized by Sherman [1980] in the case of molecular crystals, the separation of the crystal vibrations in two groups: a first one related to the internal vibrations of the
$\mathrm{SiO}_{4}$ tetrahedra and a second one related to lattice vibrations. The $\gamma_{i T}$ of the lattice modes would then be calculated with the mean bulk modulus of the lattice (in this case the crystal bulk modulus or that of the $\mathrm{Mg}-\mathrm{O}$ bond). We can then propose polyhedral Grüneisen parameters for the internal vibrations with the $\mathrm{Si}-\mathrm{O}$ bond bulk modulus (KTSi-O). The $\gamma_{i T}$ for the internal modes would thus be written as

$$
\gamma T=K T S i-O(\partial \ln v i / \partial P)
$$

The Grüneisen parameter computed with (29) and (31) agrees better with that obtained from (23) at $300 \mathrm{~K}$, but it still decreases with temperature to reach 0.86 at $2000 \mathrm{~K}$ (Table 7 ). Thus a significant improvement is obtained in this way, for the results (Table 7) agree to within $10 \%$ between 300 and $2000 \mathrm{~K}$ with $\gamma$ as given by (23). But for the moment there are no clear theoretical explanations for this procedure. It could be justified by the existence of a sublattice which undergoes most of the structural changes and in which rigid, isolated $\mathrm{SiO}_{4}$ units are embedded. In contrast, the classical approach for calculating $\gamma_{\mathrm{m}}$ from the $\gamma_{i T}$ should work for minerals having only one kind of polyhedra, or different polyhedra with similar elastic properties, of which perovskite structures are the most important examples [Williams et al., 1987; Hemley et al., 1989].

We emphasize, however, that a different explanation could be proposed for the discrepancy between Grüneisen parameters calculated with (23) and (29). Little is in fact known about the variation of $\left(\partial v_{i} / \partial P\right) T$ with temperature. The available data [Dietrich and Arndr, 1982] are sketchy and suggest that this derivative decreases with temperature, in which case the disagreement could be worse still. But moderate increases of this derivative are predicted if one assumes that vibrational frequencies are mainly determined by the volume [Richet et al., 1991], and such increases could account for the observed discrepancy at high temperatures. Measurements of $\left(\partial v_{i} / \partial P\right)_{T}$ at different temperatures are thus badly needed to assess the influence of this derivative on the various terms of (29).

In summary, the example of forsterite shows that accurate experimental $C_{p}$ data can be used to obtain reliable values of $\alpha$ and $K_{T}$ at high temperatures from the room-temperature values of these parameters and an anharmonic calculation of $C_{\nu}$. Of course, this is not possible for mantle minerals when $C_{p}$ is not known experimentally. If, as is usually the case, $\alpha$ is also unknown, then the importance of the anharmonic contributions to $C_{p}$ makes it difficult to calculate $C_{p}$ from spectroscopic data at high temperatures. In addition, the current discrepancies between Grüneisen parameters calculated from macroscopic and microscopic data also prevents reliable application of spectroscopic measurements to quantitative modeling of thermodynamic properties of mantle minerals. As pointed out above, this discrepancy requires spectroscopic measurements at both high temperature and high pressure in order to be resolved. In addition, efforts should be made to devise ways of determining the temperature dependence of thermal expansion coefficients from spectroscopic data.

Acknowledgments. We thank Fredo Vaucelle and Christophe Sotin for help in computer programing, B. Reynard for fruitful discussions, O.L. Anderson, A. Chopelas and G. Helffrich for helpful reviews. $O$. Jaoul and $Y$. Gueguen kindly provided the samples used in this study. Contribution CNRS-INSU-DBT 237. 


\section{REFERENCES}

Anderson, O.L., and I. Suzuki, Anharmonicity of three minerals at high temperature: forsterite, fayalite, and periclase, J. Geophys. Res., 88, $3549-3556,1983$

Anderson, O.L., and K. Zou, Themodynamic functions and properties of $\mathrm{MgO}$ at high compression and high temperature, J. Phys. Chem. Ref. Data, 19, 69-83, 1990.

Ashida T., S. Kume, and E. Ito, Thermodynamic aspects of phase boundary among $\alpha-, \beta$, and $\gamma-\mathrm{Mg}_{2} \mathrm{SiO}_{4}$, in High-Pressure Research in Geophysics, edited by M.H. Manghnani and Y. Syono, pp. 269-274, AGU, Washington, D.C, 1987.

Berman, R.G., and T.H. Brown, Heat capacity of minerals in the system $\mathrm{Na}_{2} \mathrm{O}-\mathrm{K}_{2} \mathrm{O}-\mathrm{CaO}-\mathrm{MgO}-\mathrm{FeO}-\mathrm{Fe}_{2} \mathrm{O}_{3}-\mathrm{Al}_{2} \mathrm{O}_{3}-\mathrm{SiO}_{2}-\mathrm{TiO}_{2}-\mathrm{H}_{2} \mathrm{O}-\mathrm{CO}_{2}$ : Representation, estimation, and high-temperature extrapolation, Contrib. Mineral. Petrol., 89, 168-183, 1985.

Besson, J.M., Pinceaux, J.P., Anastopoulos, C., and Velde, B., Raman spectra of olivine up to 65 kilobars, J. Geophys. Res., 87, 1077310775, 1982.

Boehler, R., Adiabats of quartz, coesite, olivine and magnesium oxide to $50 \mathrm{kbar}$ and $1000 \mathrm{~K}$, and the adiabatic gradient in the Earth mantle, $J$. Geophys. Res., 87, 5501-5506, 1982.

Chopelas, A., Thermochemical properties of forsterite at mantle pressures derived from vibrational spectroscopy, Phys. Chem. Miner., 17, 149$156,1990$.

Choudury, N., S.L. Chaplot, and K.R. Rao, Equation of state and melting point of forsterite, Phys. Chem. Miner., 16, 599-605, 1989.

Dietrich, P., and J. Andt, Effects of pressure and temperature on the physical behaviour of mantle-relevant olivine, orthopyroxene and garnet II, Infrared absorption and Gruneisen-parameters, in High Pressure Research in Geoscience, edited by W. Schreyer, pp. 307-309, E. Schweizerbart'sche, Stuggart, Germany, 1982.

Fei, Y., and S.K. Saxena, An equation for the heat capacity of minerals, Geochim. Cosmochim. Acta, 51, 251-254, 1987a.

Fei, Y., and S.K. Saxena, A themochemical data base for phase equilibria in the system $\mathrm{Fe}-\mathrm{Mg}-\mathrm{Si}-\mathrm{O}$ at high pressure and temperature, Phys. Chem. Miner., 13, 311-324, 19876.

Gillet, Ph., J.M. Malezieux, and M.C. Dhamelincourt, MicroRaman multichannel spectroscopy up to $2.5 \mathrm{GPa}$ using a sapphire anvil cell experimental set-up and some applications, Bull. Minéral., 111, 1-15, 1988.

Gillet, Ph., F. Guyot, and J.M. Malezieux, High pressure and high temperature Raman spectroscopy of $\mathrm{Ca}_{2} \mathrm{GeO}_{4}$ : some insights on anharmonicity, Phys. Earth Planet. Int., 58, 141-154, 1989.

Gillet, Ph., A. Le Cléac'h, and M. Madon, High-temperature Raman spectroscopy of $\mathrm{SiO}_{2}$ and $\mathrm{GeO}_{2}$ polymorphs: anharmonicity and thermodynamic properties at high-temperatures, J. Geophys. Res., 95, 21635-21655, 1990.

Grüneisen, E., Theorie des festen zustandes einatomiger elemente, Ann. Phys., 39, 257-306, 1912.

Haas, J.L., Jr, and J.R. Fisher, Simultaneous evaluation and correlation of thermodynamic data, Am. J. Sci., 276, 525-545, 1976.

Hazen, R.M., and Finger, L.W., Comparative Crystal Chemistry, John Wiley and Sons, New York, 1982.

Hemley, R.J., R.E., Cohen, A. Yeganeh-Haeri, H.K. Mao, and D.J. Weidner, Raman spectroscopy and lattice dynamics of $\mathrm{MgSiO}_{3}$ perovskite, in Perovskite: A Structure of Great Interest to Geophysics and Materials Science, Geophys. Monogr. Ser., 45, edited by A. Navrotsky and D.J. Weidner, Pp. 35-44, AGU, Washington, D.C., 1989.

Hofmeister, A.M., Single-crystal absorption and reflection infrared of forsterite and fayalite, Phys. Chem. Miner., 14, 499-513, 1987.

Hofmeister, A.M., J. Xu, H.K. Mao, P.M. Bell, and T.C. Hoering, Thermodynamics of $\mathrm{Fe}-\mathrm{Mg}$, olivines at mantle pressure: mid- and farinfrared spectroscopy at high pressure, Am. Mineral., 74, 281-306, 1989.

Isaak, D.G., O.L. Anderson, and T. Goto, Elasticity of single-crystal forsterite measured to $1700 \mathrm{~K}, J$. Geophys. Res., 94, 5895-5906, 1989.

Kajiyoshi , K., High-temperalure equation of state for mantle minerals and their anharmonic properties, M.S. Thesis, Okayama University, Okayama, Japan, 1986. (Quoted by Isaak et al. [1989]).

Kelley, K.K., Specific heats at low temperatures of magnesium orthosilicate and magnesium metasilicate, J. Am. Chem. Soc., 65, 339-341, 1943.

Kieffer. S.W.. Thermodynamics and lattice vibrations of minerals 3 ,
Lattice dynamics and an approximation for minerals with application to simple substances and framework silicates, Rev. Geophys., 17, 827849, 1979.

Kudoh, Y., and Y. Takéuchi, The crystal structure of forsterite $\mathrm{Mg}_{2} \mathrm{SiO}_{4}$ under pressure up to $149 \mathrm{kbar}, Z$. Kristallogr., 171, 291-302, 1985.

Mammone, J.F., and S.K. Shama, Pressure and temperature dependence of the Raman spectra of rutile-structure oxides, Year Book, Carnegie Inst. Washington, 78, 369-373, 1979.

McMillan, P.F., and A.M. Hofmeister, Infrared and Raman spectroscopy, in Spectroscopic Methods in Mineralogy and Geology, Reviews in Mineralogy, vol. 18, edited by F.C. Hawthome, pp. 99-159, Mineralogical Society of America, Whashington D.C. 1988.

Orr, R.L., High temperature heat contents of magnesium orthosilicate and ferrous orthosilicate, J. Am. Chem. Soc., 75, 528-529, 1953.

Price, G.D., S.C. Parker, and M. Leslie, The lattice dynamics and thermodynamics of the $\mathrm{Mg}_{2} \mathrm{SiO}_{4}$ polymorphs, Phys. Chem. Miner., 15, 181-190, 1987.

Rao, K.R., S.L. Chaplot, N. Choudury, S. Ghose, J.M. Hastings and L.M. Corliss, Lattice dynamics and inelastic neutron scattering from forsterite, $\mathrm{Mg}_{2} \mathrm{SiO}_{4}$ : phonon dispersion relation, density of states and specific heat, Phys. Chem. Miner., 16, 83-97, 1988.

Richet, P., and Y. Bottinga, Anorthite, andesine, diopside, wollastonite, cordierite and pyrope: thermodynamics of melting, glass transitions, and properties of the amorphous phases, Earth Planet. Sci. Lett., 67, 415-432, 1984

Richet, P., and G. Fiquet, High-temperature heat capacity and premelting of minerals in the system $\mathrm{CaO}-\mathrm{MgO}-\mathrm{Al}_{2} \mathrm{O}_{3}-\mathrm{SiO}_{2}, J$. Geophys. Res., $96,445-456,1991$.

Richet, P., Y.Bottinga, L. Deniélou, J.P. Petitet, and C. Téqui, Thermodynamic properties of quartz, cristobalite and amorphous $\mathrm{SiO}_{2}$ : drop calorimetry measurements between 1000 and $1800 \mathrm{~K}$ and a review from 0 to $2000 \mathrm{~K}$, Geochim. Cosmochim. Acta, 46, 2639-2658, 1982.

Richet, P., Ph. Gillet and G. Fiquet, Thermodynamic properties of minerals: macroscopic and microscopic approaches. Adv. Phys. Geochem., in press, 1991.

Robie, R.A., B.S. Hemingway, and H. Takei, Heat capacities and entropies of $\mathrm{Mg}_{2} \mathrm{SiO}_{4}, \mathrm{Mn}_{2} \mathrm{SiO}_{4}$, and $\mathrm{Co}_{2} \mathrm{SiO}_{4}$ between 5 and $380 \mathrm{~K}, \mathrm{Am}$. Mineral., 67, 470-482, 1982.

Saxena, S.K., Assessment of thermal expansion, bulk modulus, and heat capacity of enstatite and forsterile. J. Phys. Chem. Solids, 49, 1233$1235,1988$.

Saxena, S.K., Assessment of bulk modulus, thermal expansion and heat capacity of minerals, Geochim. Cosmochim. Acta, 53, 785-789, 1989.

Sherman, W.F., Bond anharmonicities, Grüneisen parameters and pressure-induced shifts, J. Phys. C Solid State. Phys., 13, 4601-4613, 1980.

Slater, J.C., Introduction to Chemical Physics, 521 pp, McGraw Hill, New York, 1939.

Sotin, C., Contribution à l'étude de la structure et de la dynamique inteme des planètes, Thèse, 395 pp., Université de Paris VII, 1986.

Suzuki, I., O.L. Anderson, and Y. Sumino, Elastic properties of a singlecrystal forsterite $\mathrm{Mg}_{2} \mathrm{SiO}_{4}$, up to $1200 \mathrm{~K}$, Phys. Chem. Miner, , 10, $38-46,1983$.

Tarantola, A., and B. Valette, Generalized non-linear inverse problems solved using the least square criterion, Rev. Geophys., 20, 219-232, 1982.

Watanabe, H., Thermochemical properties of synthetic high-pressure compounds relevant to the Earth's mantle, in High-Pressure Research in Geophysics, edited by S. Akimoto and M.H. Manghnani, pp. 441-464, D. Reidel, Hingham Mass., 1982.

White, G.K., R.B. Roberts, and J.G. Collins, Thermal properties and Grüneisen functions of forsterite, $\mathrm{Mg}_{2} \mathrm{SiO}_{4}$, High Temp. High Pressures, 17, 61-66, 1985.

Williams, Q., R. Jeanloz, and P.F. MacMillan, Vibrational spectrum of $\mathrm{MgSiO}_{3}$ perovskite: zero pressure Raman and mid-infrared spectra to $27 \mathrm{GPa}$, J. Geophys. Res., 92, 8116-8128, 1987.

G. Fiquet and $\mathrm{Ph}$. Gillet, Laboratoire de Minéralogie Physique, CAESS (UPR 4661), Université de Rennes I, 35042 Rennes Cedex, France.

F. Guyot and P. Richet, Département des Géomatériaux, Institut de Physique du Globe, 4 place Jussieu, 75005 Paris, France.

(Received November 5, 1990;

revised January 23, 1991;

accepted February 28, 1991.) 